\title{
Spectral BRDF-based determination of proper measurement geometries to characterize color shift of special effect coatings
}

\author{
Alejandro Ferrero, ${ }^{1, *}$ Ana Rabal, ${ }^{1}$ Joaquín Campos, ${ }^{1}$ Francisco Martínez-Verdú, ${ }^{2}$ Elísabet Chorro, ${ }^{2}$ \\ Esther Perales, ${ }^{2}$ Alicia Pons, ${ }^{1}$ and María Luisa Hernanz ${ }^{1}$ \\ ${ }^{1}$ Instituto de Óptica, Consejo Superior de Investigaciones Científicas (CSIC), Madrid 28006, Spain \\ ${ }^{2}$ Department of Optics, Pharmacology and Anatomy, University of Alicante, Alicante 03080, Spain \\ *Corresponding author: alejandro.ferrero@csic.es
}

Received September 14, 2012; revised December 5, 2012; accepted December 21, 2012; posted December 21, 2012 (Doc. ID 176060); published January 11, 2013

\begin{abstract}
A reduced set of measurement geometries allows the spectral reflectance of special effect coatings to be predicted for any other geometry. A physical model based on flake-related parameters has been used to determine nonredundant measurement geometries for the complete description of the spectral bidirectional reflectance distribution function (BRDF). The analysis of experimental spectral BRDF was carried out by means of principal component analysis. From this analysis, a set of nine measurement geometries was proposed to characterize special effect coatings. It was shown that, for two different special effect coatings, these geometries provide a good prediction of their complete color shift. (c) 2013 Optical Society of America
\end{abstract}

OCIS codes: $\quad 330.1710,330.1720,290.1483$

\section{INTRODUCTION}

Coatings with special-effect pigments [1] create the illusion of optical depth and present an eye-catching effect of angledependent color. This color shift occurs not only in lightness and in chroma but also, unlike metallic coatings, in hue. As a consequence of their appealing appearance, these coatings have become very popular in the automotive industry [2], and they are widely used in other markets, such as the cosmetics industry or, for a more functional purpose, anticounterfeit measures in currency.

This kind of coating consists of a transparent substrate having embedded flakes of special-effect pigments that are horizontally arranged. Its layered structure gives rise to interferences, which is the origin of this special perceived effect [3-5]. These coatings are usually modeled as a clear coat and a base coat, which contains both special-effect and absorption pigments. The specular component of the reflection is produced mainly in the clear coat, and both the diffuse reflection and the hue variation are produced in the base coat.

The increasing popularity of these coatings demands the development of new techniques and instruments to characterize the spectral reflectance and the color as a function of the different irradiation/viewing geometrical configurations [3,6-14], and for different illuminants and real light sources [15]. Full knowledge of the spectral bidirectional reflectance distribution function (BRDF) of these coatings makes it possible to infer the coating color for any geometrical configuration (irradiation and viewing directions and solid angles) as well as the spectral distribution of the irradiation [16]. The problem to be faced is that, given the complex spectral reflectance that these coatings have, it is not clear which measurement geometries should be used in order to fully characterize them. The study of Takagi et al. [17] concluded that almost
2000 geometries would be required; however, nowadays it is an unrealizable goal for most industries due to time constraints and cost-efficiency reasons. The ASTM standard for multiangle color measurement of interference pigments [18] recommends eight uniplanar measurement geometries for the quality control application, but it points out that, for other applications, additional geometries are "desirable or needed." In this context, Nadal and Early [3] proposed a combination of three irradiation angles and five aspecular angles for the adequate colorimetric characterization of these coatings.

The aim of this work is to find a reduced set of measurement geometries that will allow us to predict the spectral reflectance (and therefore, the color) for any other geometry, regardless of the special effect coating. This, in turn, should help manufacturers to design instruments that lead to a more accurate characterization of the spectral reflectance of special effect coatings, and it should provide some potential applications in optical formulation and digital rendering for computer graphics. The specular geometries to characterize the gloss were excluded.

The procedure that was followed to reduce the number of measurement geometries is based on the physical interpretation recently used by Kirchner and Cramer [19]. They proposed a new perspective to better understand the spectral reflectance for different geometries: Instead of using the conventional experimental parameters (irradiation and viewing angles), they opted to include flake-related ones, such as flake orientation and incident angle on the flake. Then, a model based on this perspective was used to determine which measurement geometries were redundant for the complete description of the spectral BRDF. The analysis of experimental spectral BRDF data was made without prior colorimetric simplification: the variation across the different 
geometries of colorimetric values, such as lightness, hue, or chroma had not been not previously studied; only the variation of the corresponding set of BRDF spectra had been analyzed from a strictly optical and mathematical point of view. In order to accomplish this multivariate task, principal components analysis (PCA) was used, whose ability to extract patterns out of this kind of data had been already proven in previous works [20,21]. PCA distinctly separates the spectral variable (wavelength) from the geometrical variables (spherical coordinates of the irradiation and viewing directions) by decomposing the spectral BRDF in decorrelated spectral components.

The measurement of the BRDF was performed using the GEFE gonio-spectrophotometer [22], which was developed at the Spanish Council for Scientific Research's Optics Institute (IO-CSIC). It consists of a fixed, uniform, and collimated light source and a robot-arm that places the sample, making it possible to change automatically and simultaneously the irradiation direction (defined by means of the spherical coordinates $\theta_{i}$ and $\left.\phi_{i}\right)$ and the viewing one $\left(\theta_{s}, \phi_{s}\right)$. These spherical coordinates are defined relative to the Sample Coordinate System, whose $z$ axis is parallel to the sample's normal direction (Figure 1). For the data acquisition, a CS-2000 A Konica Minolta spectroradiometer was employed. This device operates within the visible range [380-780 nm], performing spectral measurements at a $1 \mathrm{~nm}$ sampling interval and having a $4 \mathrm{~nm}$ bandwidth.

\section{FLAKE-BASED MODEL}

Effect pigment coatings consist of a base coat (including binder, metallic, or dielectric flake-shaped pigments and absorption pigments) and a clear coat [5]. Since the color travel (or spectral-reflectance variation) of these coatings is mainly due to the reflection of the light on the flakes, the characteristics of this reflection must be fully understood.

Following the approach suggested by Kirchner and Cramer [19], the flake-related reflection phenomenon is modeled as shown in Fig. 2. The incoming light with an incidence angle $\theta_{i}$ relative to the sample's normal is refracted at an angle $\theta_{i}^{\prime}$, is transmitted through the binder according to Snell's law, and impinges upon the flake at an incidence angle $\theta_{\text {inc }}$ with respect to the flake's normal. The flake is assumed to be completely flat. The signs of both $\theta_{i}$ and $\theta_{\text {inc }}$ are positively defined. If the flake is metallic, the spectral distribution of the reflected light will not depend on $\theta_{\text {inc }}$, whereas if the flake is

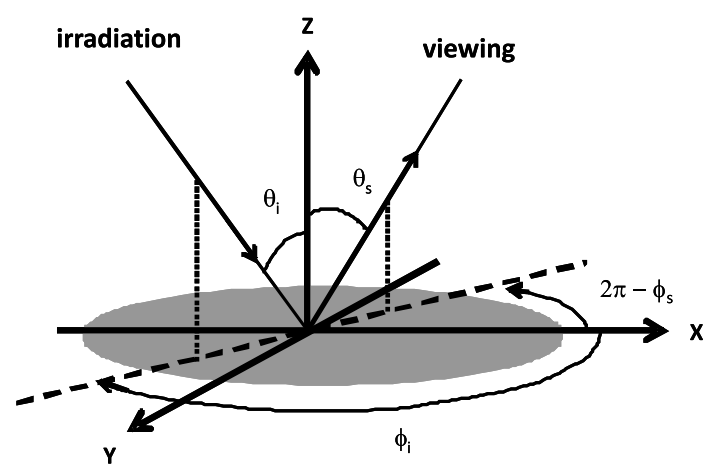

Fig. 1. Sample coordinate system, defined by the set of spherical coordinates for the irradiation $\left(\theta_{i}, \phi_{i}\right)$ and the viewing directions $\left(\theta_{s}, \phi_{s}\right)$.

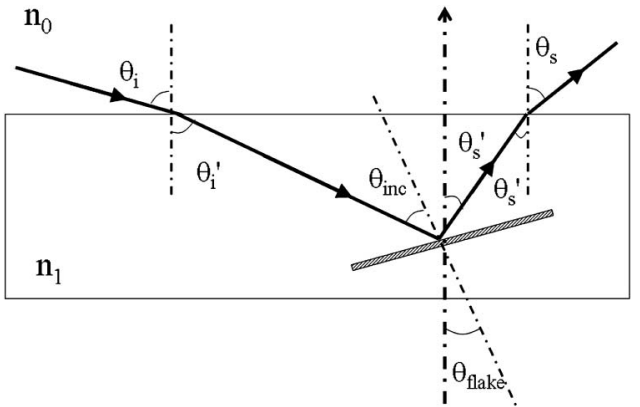

Fig. 2. Geometrical variables needed to describe the flake-related reflection mechanism.

dielectric the reflection's spectrum will be angle dependent due to the interference occurring within the flake, as has been well explained in the literature [23]. This is the reason why, when the irradiation and/or viewing geometry changes, the perceived hue varies much more dramatically in the case of special-effect pigment coatings (dielectric flakes) than with effect pigment coatings (metallic flakes). For a given $\theta_{i}$, the angle $\theta_{\text {inc }}$ is not constant, but it will depend on the flake's disorientation angle $\theta_{\text {flake }}$ of that particular flake; that is, the angle between the sample's normal and the flake's normal. The sign of $\theta_{\text {flake }}$ is positively defined when $\theta_{\text {inc }}$ is smaller than $\theta_{i}^{\prime}$, and negatively defined otherwise. The light is reflected at the flake's surface at an angle $\theta_{s}^{\prime}$ with respect to the sample's normal, transmitted again through the binder, and refracted at the interface an angle $\theta_{s}$, which is the measurement's viewing angle. For a calculation of the flake parameters, the reader may refer to the literature [19].

According to this description, for a given pair $\left(\theta_{i}, \theta_{s}\right)$, there is a unique pair $\left(\theta_{\text {inc }}, \theta_{\text {flake }}\right)$ that reproduces that same geometry. This means that the description in terms of the measurement geometry pair $\left(\theta_{i}, \theta_{s}\right)$, which refers to the sample's surface, can be replaced by a description given in terms of the pair $\left(\theta_{\text {inc }}, \theta_{\text {flake }}\right)$, which refers to equivalent flakes having the same disorientation angle and irradiated with the same incidence angle.

There are two main reasons that drive us to use this alternative terminology or parameter set. First, $\theta_{\text {inc }}$, unlike $\theta_{i}$, unequivocally determines the phase difference that is responsible for the interference phenomenon occurring within the flake and, consequently, for the spectral distribution of the light reflected at the flake. Second, $\theta_{\text {flake }}$ is quite linked to the aspecular angle ( $\theta_{\text {asp }}$, angular difference between the specular angle and the viewing angle). If $\theta_{\text {flake }}$ is zero, the viewing is done in specular conditions; if $\theta_{\text {flake }}$ is negative, the viewing lies on what is known in the literature as transgeometry (negative aspecular angle); and if $\theta_{\text {flake }}$ is positive, the viewing lies on what is known as cisgeometry (positive aspecular angle) [19]. The relation between $\theta_{\text {flake }}$ and $\theta_{\text {asp }}$ for 648 measurement geometries (with $\theta_{i}$ and $\theta_{s}$ ranging from $0^{\circ}$ to $85^{\circ}$ at steps of $5^{\circ}$, for $\phi_{s}=0^{\circ}$ and $180^{\circ}$ ) is shown in Fig. 3 (the binder's refractive index is assumed to be 1.5 , which is correct for most effect-coatings [19]). A particular value of $\theta_{\text {asp }}$ corresponds approximately to a value of $\theta_{\text {flake }}$ within a $\pm 3.5^{\circ}$ range. Therefore, even if $\theta_{i}$ varies, as long as $\theta_{\text {asp }}$ is kept constant, the radiance reflected from the flakes will always come from flakes having approximately the same disorientation. In other words, regardless of the irradiation angle, the viewer is always looking at the same group of flakes as long as $\theta_{\text {asp }}$ does not vary. 


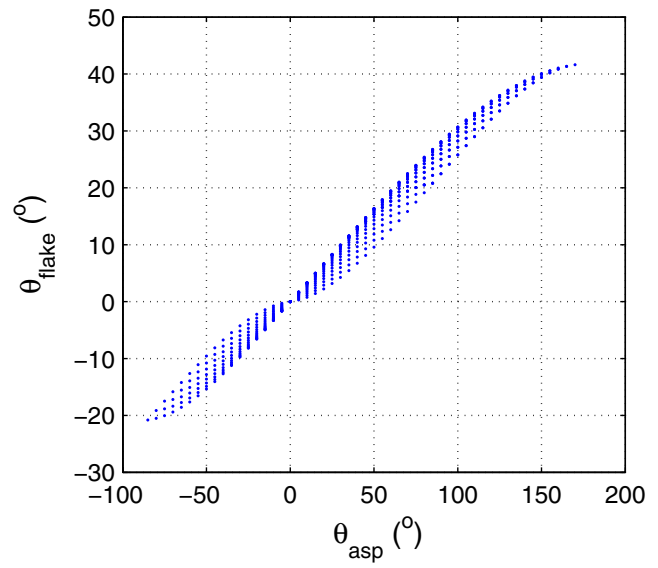

Fig. 3. (Color online) Relation between $\theta_{\text {flake }}$ and $\theta_{\text {asp }}$ for 648 measurement geometries $\left(\theta_{i}\right.$ and $\theta_{s}$ range from $0^{\circ}$ to $85^{\circ}$ at steps of $5^{\circ}$ ), for $\phi_{s}=0^{\circ}$ and $180^{\circ}$.

Since flake disorientation distributions $D\left(\theta_{\text {flake }}\right)$ usually show a peak for the horizontal orientation $\left(\theta_{\text {flake }}=0^{\circ}\right.$; that is, flakes lying parallel to the sample's surface), the reflected radiation will peak for the specular direction. On the other hand, $D\left(\theta_{\text {flake }}\right)$ is expected to be almost Gaussian [24], a fact that should affect the $\theta_{s}$-dependence of the reflected amplitude, as shown in Fig. 4 . However, the measurement geometry pair $\left(\theta_{i}, \theta_{s}\right)$ is better suited than $\left(\theta_{\text {inc }}, \theta_{\text {flake }}\right)$ to determine the optical path length of the light travelling through the binder (see Fig. 2). The higher $\theta_{i}$ or $\theta_{s}$, the longer the optical path length and the more exposed the light is to the absorption pigments. Its does not mean that the color of the absorption pigments is better observed in those conditions, but that the spectral distribution of the light is more modified by the spectral scattering of the absorption pigments than at lower $\theta_{i}$ or $\theta_{s}$ angles. The color of the absorption pigments will be more visible when the reflected light on special effect pigments is not the dominant contribution to the total refection, that is, for high $\theta_{\text {flake }}$ angles.

According to our interpretation, perhaps the most important conclusions that can be drawn from this approach are:

- $\theta_{\text {inc }}$ determines the spectral distribution of the reflection at the flakes and, therefore, has a marked influence on the change of the perceived hue.

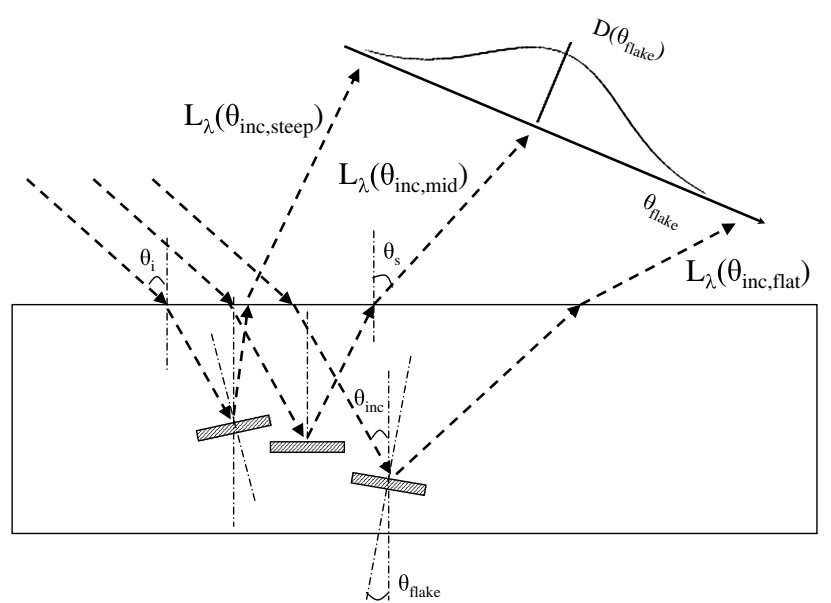

Fig. 4. Scattering of the optical radiation due to the disorientation of the flakes. $L_{\lambda}$ is the spectral radiance.
- $\theta_{\text {flake }}$ determines the amplitude of the flake-related reflection for the corresponding aspecular angle and, therefore, it has a significant influence on the change of chroma and lightness.

These two conclusions are directly linked to the interpretation of the broadly used concepts of interference and aspecular lines, which have been revealed to be quite important to identify samples within a CIELAB representation [19]:

- An interference line is the locus of those measurement geometries having a constant $\theta_{\text {asp }}$. In this case, only $\theta_{i}$ varies, with the corresponding variation of $\theta_{\text {inc }}$ and, consequently, of the spectral distribution of its reflection when interference takes place. Therefore, an interference line mainly shows the change in the spectral distribution produced by the flakes at an almost constant amplitude (constant $\theta_{\text {asp }}$ ). In color terms, these lines tend to show an important hue shift. Across a specific interference line, the range of possible $\theta_{\text {inc }}$ angles depends on the $\theta_{\text {flake }}$ angle of the observed flakes. The higher the $\theta_{\text {flake }}$ angle (or the aspecular angle), the smaller the maximum achievable $\theta_{\text {inc. }}$. For instance, the manifestation of the interference at steep $\theta_{\text {inc }}$ angles is higher at trans- than at cis-geometries.

- An aspecular line is the locus of those measurement geometries having a constant $\theta_{i}$. The higher $\theta_{i}$ for a given aspecular line, the higher the average $\theta_{\text {inc }}$ angle. Consequently, for an aspecular line at high $\theta_{i}$, the change in the spectral distribution due to interference occurring at the flakes at high $\theta_{\text {inc }}$ values will be observed and, equivalently, for low $\theta_{i}$ angles. Therefore, across an aspecular line, the hue change is small compared to the chroma or lightness changes. Finally, it should be pointed out that, at some value of the aspecular angle, the contribution to the reflected radiation from the flakes will begin to be negligible relative to other diffuse contributions coming from the binder, the pigmented base coat, or the clear coat.

Bearing in mind these considerations, we propose an approximation for the spectral BRDF $\left[f_{r}(\lambda)\right]$ of this kind of coating as the sum of two components: $f_{r \text {,eff }}(\lambda)$, representing the gonio-apparent reflection produced by the special-effect pigments, and $f_{r, 0}(\lambda)$, representing the completely or partially diffuse reflection produced by the usual mechanisms:

$$
\begin{aligned}
f_{r}\left(\theta_{i}, \phi_{i} ; \theta_{s}, \phi_{s} ; \lambda\right)= & f_{r, \mathrm{eff}}\left(\theta_{i}, \phi_{i} ; \theta_{s}, \phi_{s} ; \lambda\right) \\
& +f_{r, 0}\left(\theta_{i}, \phi_{i} ; \theta_{s}, \phi_{s} ; \lambda\right) .
\end{aligned}
$$

$f_{r, \text { eff }}$ is computed by adding up the contributions of the reflections at the individual flakes. Therefore

$$
f_{r, \text { eff }}\left(\theta_{i}, \phi_{i} ; \theta_{s}, \phi_{s} ; \lambda\right)=D\left(\theta_{\text {flake }}\right) f_{r, \text { lake }}\left(\theta_{i}, \phi_{i} ; \theta_{s}, \phi_{s} ; \lambda\right)
$$

where $D\left(\theta_{\text {flake }}\right)$ is the disorientation distribution of the flakes and $f_{r, \text { flake }}\left(\theta_{i}, \phi_{i} ; \theta_{s}, \phi_{s} ; \lambda\right)$ is the contribution of a single flake to the total BRDF, which, in its turn and based on the definition of the BRDF (radiance of the reflected optical radiation by incident irradiance), can be expressed as follows:

$$
f_{r, \text { flake }}\left(\theta_{i}, \phi_{i} ; \theta_{s}, \phi_{s} ; \lambda\right)=\frac{T\left(\theta_{i}, \lambda\right) R_{\text {flake }}\left(\theta_{\mathrm{inc}}, \lambda\right) T\left(\theta_{s}, \lambda\right)}{\Omega_{s}\left(\theta_{s}, \phi_{s}\right)}
$$


where $R_{\text {flake }}\left(\theta_{\text {inc }}, \lambda\right)$ is the spectral reflectance of a flake (which depends on the light's incidence angle, $\left.\theta_{\text {inc }}\right), T(\theta, \lambda)$ is the spectral transmittance of the medium, consisting of binder and absorption pigments (it depends on $\theta_{i}$ when the optical radiation travels to the flake and on $\theta_{s}$ when it travels away from the flake), and $\Omega_{s}\left(\theta_{s}, \phi_{s}\right)$ is the projected viewing angle.

\section{DETERMINATION OF THE MEASUREMENT GEOMETRIES}

\section{A. PCA Approach}

According to this model, the functions $D\left(\theta_{\text {flake }}\right)$ and $R_{\text {flake }}\left(\theta_{\text {inc }}, \lambda\right)$ model the effect due to the addition of the flakes. Therefore, to characterize special effect coatings, we will choose as measurement geometries those that provide enough information about these two functions.

In a previous paper [21], the authors showed that, using a procedure based on PCA, it was possible to express the spectral BRDF of a special-effect pigment coating as a sum of components, in such a way that spectral and geometrical variables are separated:

$$
\begin{aligned}
f_{r}\left(\theta_{i}, \phi_{i} ; \theta_{s}, \phi_{s} ; \lambda\right)= & \left\langle f_{r}\left(\theta_{i}, \phi_{i} ; \theta_{s}, \phi_{s}\right)\right\rangle_{\lambda} \\
& \times\left[1+\sum_{j=1}^{M} c_{j}\left(\theta_{i}, \phi_{i} ; \theta_{s}, \phi_{s}\right) H_{j}(\lambda)\right] .
\end{aligned}
$$

This equation is made up of two factors: the spectral average of $f_{r}\left(\left\langle f_{r}\right\rangle_{\lambda}\right)$ having just a geometrical dependence, and the sum of $M+1$ addends or components (shown inside square brackets), where the first one is equal to one. Every addend is factorized into two: one containing the spectral information, $H_{j}(\lambda)$ (having an average value of 0 and a standard deviation of 1), and another factor containing the geometrical information, $c_{j}\left(\theta_{i}, \phi_{i} ; \theta_{s}, \phi_{s}\right)$, which can be regarded as the weight of the spectral distribution $H_{j}(\lambda)$ at a given geometrical configuration.

In the previous paper mentioned above [21], the measurement and representation of the BRDF of the special-effect pigment material Colorstream T20-02 WNT Arctic Fire was thoroughly discussed [25]. The spectral BRDF was sampled at 448 different measurement geometries, each one of them corresponding to a specific combination of $\theta_{i}$ and $\theta_{s}$ (which take values ranging from $0^{\circ}$ to $70^{\circ}$ at steps of $10^{\circ}$ ), $\phi_{i}$ (which takes only the value $0^{\circ}$ ), and $\phi_{s}$ (which takes values ranging from $0^{\circ}$ to $180^{\circ}$, at steps of $30^{\circ}$ ).

It was found that just four components $H_{j}(\lambda)$ account for $99.4 \%$ of the data's total relative variance. $H_{1}$ has a very small variation though all the geometries and, in combination with the poorly contributing $\mathrm{H}_{4}$, it may be related to the absorption pigments. Since the weights $c_{j}$ of the components $\mathrm{H}_{2}$ and $\mathrm{H}_{3}$ do vary most with the viewing angle $\theta_{s}$, they should be mainly responsible for the hue changes observed on the sample (special-effect appearance), produced by the interference occurring at the interference pigments (see Fig. 5). The relation between the two weights $c_{j}$ associated with the interference pigments should vary for low aspecular angles, whereas for high ones they should not change too significantly. The weight $c_{2}$ dominates over $c_{3}$ at low $\theta_{i}$, but $c_{3}$ dominates over $c_{2}$ at high $\theta_{i}$. Therefore, the component $\mathrm{H}_{2}$ should be related to the spectral reflectance of the flake at low $\theta_{\text {inc }}$, and $H_{3}$ to the spectral reflectance of the flake at high $\theta_{\text {inc }}$. More importantly, it seems
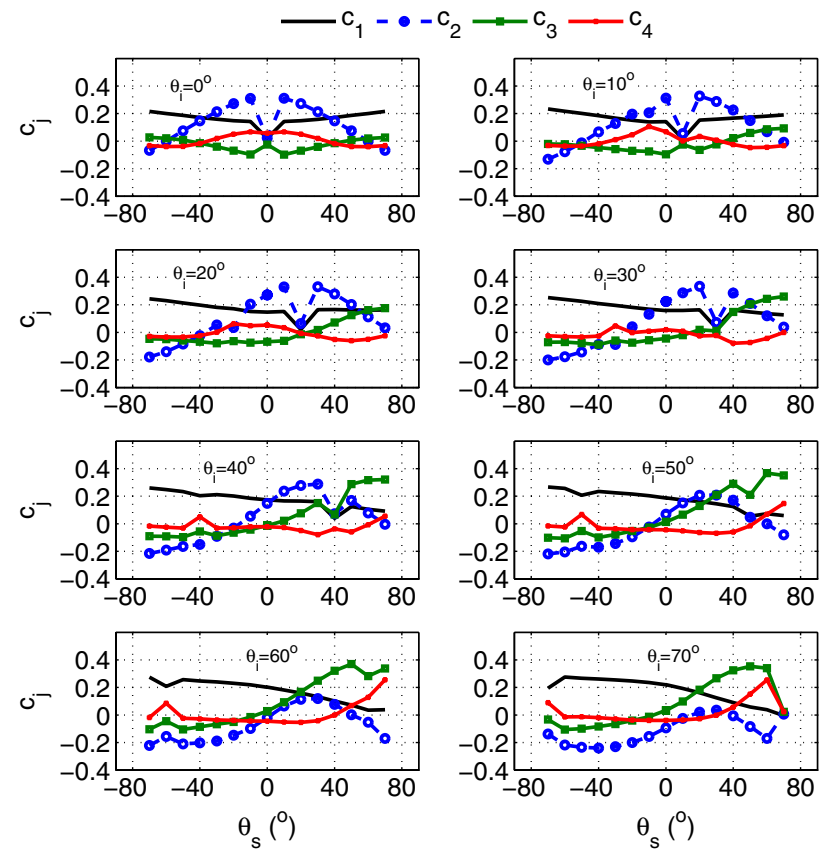

Fig. 5. (Color online) Weights $c_{j}$ as a function of the viewing angle $\theta_{s}$ for different in-plane geometrical configurations corresponding, respectively, to $H_{1}, H_{2}, H_{3}$, and $H_{4}$. Positive and negative $\theta_{s}$ indicate $\phi_{s}=180^{\circ}$ and $0^{\circ}$, respectively (Arctic Fire). This figure corresponds to Fig. 4 in [21].

that all intermediate spectral reflectances can be simply expressed as a linear combination of both components. This conclusion would allow $R_{\text {flake }}$ to be written as

$$
R_{\text {flake }}\left(\theta_{\text {inc }}, \lambda\right)=a_{\text {low }}\left(\theta_{\text {inc }}\right) R_{\text {low }}(\lambda)+a_{\text {high }}\left(\theta_{\text {inc }}\right) R_{\text {high }}(\lambda),
$$

where $R_{\text {low }}$ and $R_{\text {high }}$ are, respectively, the spectral reflectances of the flakes at low and high $\theta_{\text {inc }}$; whereas $a_{\text {low }}$ and $a_{\text {high }}$ are their corresponding weights.

Once the spectral BRDF is expressed as described in Eq. (4), this equation should be connected to Eqs. (1)-(3), and $(5)$ in order to assess which measurement geometries are required to fully characterize the functions $D\left(\theta_{\text {flake }}\right)$ and $R_{\text {flake }}\left(\theta_{\text {inc }}, \lambda\right)$.

The main problem is that the PCA result cannot be entirely related to the physical model, since PCA yields the weights of the components when they are added around the mean value $\left\langle f_{r}\left(\theta_{i}, \phi_{i} ; \theta_{s}, \phi_{s}\right)\right\rangle_{\lambda}$, and this is the reason why, unlike the flakebased physical model, it is possible to obtain negative addends.

B. Integration of the PCA Result into the Physical Model The physical model comprising Eqs. (1)-(3), and (5) can be rewritten very similarly to Eq. (4):

$$
\begin{aligned}
& f_{r, m}\left(\theta_{i}, \phi_{i} ; \theta_{s}, \phi_{s} ; \lambda\right) \\
& \quad=\left\langle f_{r, m}\left(\theta_{i}, \phi_{i} ; \theta_{s}, \phi_{s}\right)\right\rangle_{\lambda} \sum_{j=1}^{3} c_{j, m}\left(\theta_{i}, \phi_{i} ; \theta_{s}, \phi_{s}\right) H_{j, m}(\lambda),
\end{aligned}
$$

where

$$
c_{1, m}\left(\theta_{i}, \phi_{i} ; \theta_{s}, \phi_{s}\right)=\frac{\left\langle f_{r, 0}\left(\theta_{i}, \phi_{i} ; \theta_{s}, \phi_{s}\right\rangle_{\lambda}\right.}{\left\langle f_{r, m}\left(\theta_{i}, \phi_{i} ; \theta_{s}, \phi_{s}\right)\right\rangle_{\lambda}},
$$




$$
\begin{gathered}
c_{2, m}\left(\theta_{i}, \phi_{i} ; \theta_{s}, \phi_{s}\right)=\frac{D\left(\theta_{\text {flake }}\right) a_{\text {low }}\left(\theta_{\text {inc }}\right) T\left(\theta_{i}\right) T\left(\theta_{s}\right)}{\left\langle f_{r, m}\left(\theta_{i}, \phi_{i} ; \theta_{s}, \phi_{s}\right)\right\rangle_{\lambda} \Omega_{s}\left(\theta_{s}, \phi_{s}\right)}, \\
c_{3, m}\left(\theta_{i}, \phi_{i} ; \theta_{s}, \phi_{s}\right)=\frac{D\left(\theta_{\text {flake }}\right) a_{\text {high }}\left(\theta_{\text {inc }}\right) T\left(\theta_{i}\right) T\left(\theta_{s}\right)}{\left\langle f_{r, m}\left(\theta_{i}, \phi_{i} ; \theta_{s}, \phi_{s}\right)\right\rangle_{\lambda} \Omega_{s}\left(\theta_{s}, \phi_{s}\right)}, \\
H_{1, m}(\lambda)=f_{r, 0}(\lambda), \\
H_{2, m}(\lambda)=R_{\text {low }}(\lambda) T^{2}(\lambda), \\
H_{3, m}(\lambda)=R_{\text {high }}(\lambda) T^{2}(\lambda),
\end{gathered}
$$

and $T$ and $f_{r_{0}}$ are both factorized in a spectral term and a nonspectral term $\left(\langle\rangle_{\lambda}\right)$. For instance, $T=T(\lambda) \times$ $\left\langle T\left(\theta_{i}, \phi_{i} ; \theta_{s}, \phi_{s}\right)\right\rangle_{\lambda}$.

The first addend is an important difference between Eqs. (4) and (6). This is so because PCA has to be performed on average-subtracted data, resulting in $H_{j}(\lambda)$ spectra having average 0 (positive and negative values) and negative $c_{j}$ values for some geometries, unlike $H_{j, m}(\lambda)$ and $c_{j, m}$, whose values are positively defined. However, both $c_{j}$ and $c_{j, m}$ could represent the same thing (see Fig. 5): The proportion of a well-defined spectral shape $\left(H_{j}\right.$ or $\left.H_{j, m}\right)$ at specific geometries.

An important result obtained from the interpretation of the weights $c_{j}$ from PCA is shown in Fig. $\underline{6}$. The evolution of $c_{2}-c_{2, \min }$ as a function of $\theta_{\text {flake }}$ is plotted for $0^{\circ}<\theta_{\text {inc }} \leq 7^{\circ}$, $7^{\circ}<\theta_{\text {inc }} \leq 10^{\circ}, \quad 10^{\circ}<\theta_{\text {inc }} \leq 25^{\circ}$, and $25^{\circ}<\theta_{\text {inc }} \leq 35^{\circ}$. A Gaussian curve can be fitted to these data as long as the $\theta_{\text {inc }}$ values are not too high $\left(<25^{\circ}\right)$. The fitting curve was called $d\left(\theta_{\text {flake }}\right)$. Therefore, $c_{2}$ behaves in this case as expected for the weight $c_{2, m}$ of the physical model, when $a_{\text {low }}$ is constant for a range of low $\theta_{\text {inc }}$ angles; that is, a Gaussian function of $\theta_{\text {flake }}$, similarly to $D\left(\theta_{\text {flake }}\right)$. Therefore, it can be stated that there is a certain connection between the PCA result and the proposed theoretical model. It should be noticed that the factor $T\left(\theta_{i}\right) T\left(\theta_{s}\right) /\left(\left\langle f_{r, m}\right\rangle_{\lambda} \times \Omega_{s}\right)$ in Eqs. (ㅁ) and (9) is a

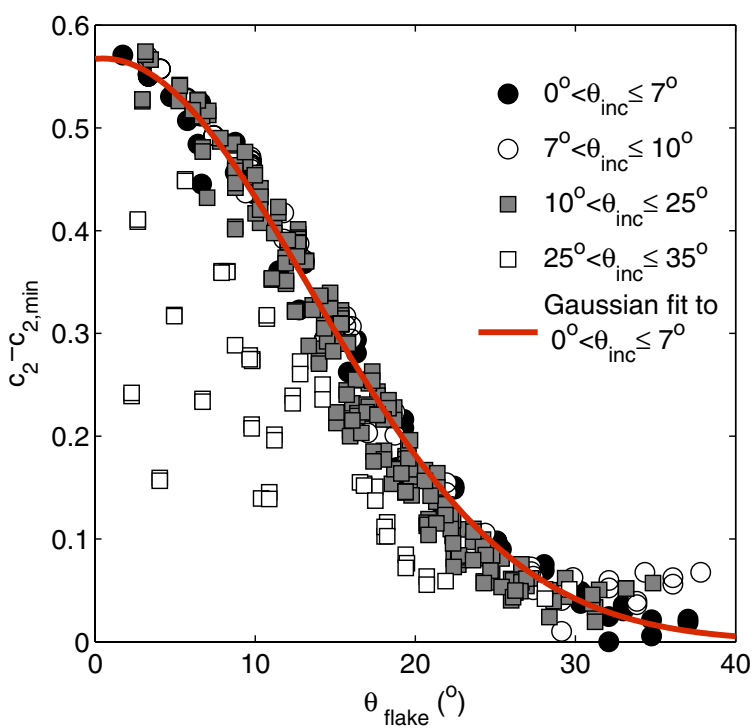

Fig. 6. (Color online) Relation of the weights of $H_{2}$ with $\theta_{\text {flake }}$ for $0^{\circ}<\theta_{\text {inc }} \leq 7^{\circ}, 7^{\circ}<\theta_{\text {inc }} \leq 10^{\circ}, 10^{\circ}<\theta_{\text {inc }} \leq 25^{\circ}$, and $25^{\circ}<\theta_{\text {inc }} \leq 35^{\circ}$. constant when the BRDF is dominated by the reflectance on the flakes $\left(\left\langle f_{r}\right\rangle_{\lambda} \sim\left\langle f_{\text {eff }}\right\rangle_{\lambda}\right)$.

Once it is known that a connection can be established between the PCA weights and $\theta_{\text {flake }}$, it is straightforward to find a relation with $\theta_{\text {inc }}$, which would provide information about the spectral reflectance of the flake for every geometry. The derived function $d\left(\theta_{\text {flake }}\right)$ was used to horizontally "orientate" (in a mathematical way) all the flakes, by dividing minimumsubtracted values of $c_{2}$ and $c_{3}$ by $d\left(\theta_{\text {flake }}\right)$ ( $a_{\text {low }, p}$ and $a_{\text {high }, p}$, respectively):

$$
\begin{gathered}
a_{\text {low }, p}=\frac{c_{2}-c_{2, \mathrm{~min}}}{d\left(\theta_{\text {flake }}\right)}, \\
a_{\text {high }, p}=\frac{c_{3}-c_{3, \mathrm{~min}}}{d\left(\theta_{\text {flake }}\right)} .
\end{gathered}
$$

The values of $a_{\mathrm{low}, p}$ and $a_{\text {high, } p}$ are shown in Fig. 7 , but only for $\theta_{\text {flake }}<7^{\circ}$, which is the range where the special effect is of higher importance (low $\theta_{\text {asp }}$ ) and, consequently, less noisy for the characterization. Again, it is observed how the result yielded by PCA can be connected to the expected behavior of the proposed model [ $a_{\text {low }}$ and $a_{\text {high }}$ in Eq. (5)]: Simply, the lower $\theta_{\text {inc }}$ is on the flake, the higher the contribution of the spectral distribution $R_{\text {low }}(\lambda)$, whereas $R_{\text {high }}(\lambda)$ dominates at high $\theta_{\text {inc }}$ angles.

The main conclusion of this assessment is that, from a multivariate analysis such as PCA, it is possible to express the special effect of the pigments as

$$
\begin{aligned}
f_{\text {eff }, \text { pca }}\left(\theta_{i}, \phi_{i} ; \theta_{s}, \phi_{s} ; \lambda\right)= & d\left(\theta_{\text {flake }}\right)\left[a_{\text {low }, p} H_{\text {low }}(\lambda)\right. \\
& \left.+a_{\text {high }, p} H_{\text {high }}(\lambda)\right],
\end{aligned}
$$

where $H_{\text {low }}(\lambda)$ and $H_{\text {high }}(\lambda)$ are the eigenspectra from PCA whose weights increase at low and high $\theta_{\text {inc }}$, respectively. It is important to point out that this separation of variables is possible in terms of the pair $\left(\theta_{\text {inc }}, \theta_{\text {flake }}\right)$, but not in terms of the pair $\left(\theta_{i}, \theta_{s}\right)$.

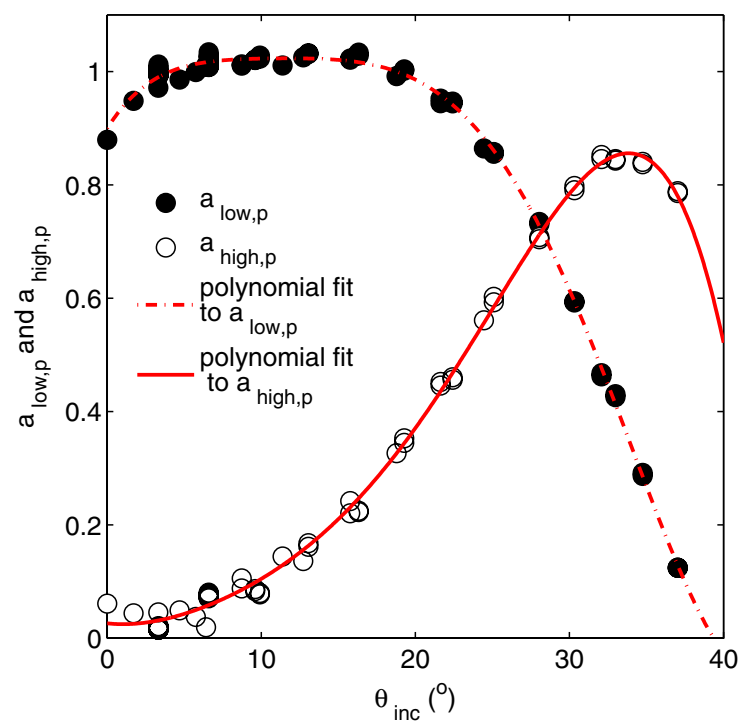

Fig. 7. (Color online) Relative weights $a_{\text {low. } p}$ and $a_{\text {high. } p}$ as a function of the incidence angle on the flake. In this case, sixth-degree polynomials were fitted to the experimental points. 
Table 1. Proposal for the Optimal Measurement Geometries for Predicting the Color Appearance Variance of Special Effect Coatings

\begin{tabular}{|c|c|c|c|c|c|c|c|}
\hline & $\left(\theta_{\text {inc }}, \theta_{\text {flake }}\right)$ pair & $\theta_{i}\left({ }^{\circ}\right)$ & $\theta_{s}\left({ }^{\circ}\right)$ & $\phi_{s}-\phi_{i}\left(^{\circ}\right)$ & $\theta_{\text {inc }}\left({ }^{\circ}\right)$ & $\theta_{\text {flake }}\left({ }^{\circ}\right)$ & $\theta_{\text {asp }}\left({ }^{\circ}\right)$ \\
\hline 1 & $\left(\sim 15^{\circ},<7^{\circ}\right)$ & 20 & 30 & 180 & 16.3 & 3.1 & -10 \\
\hline 2 & $\left(\sim 10^{\circ},<7^{\circ}\right)$ & 20 & 10 & 180 & 10 & 3.3 & 10 \\
\hline 3 & $\left(<7^{\circ},<7^{\circ}\right)$ & 20 & 0 & $0 / 180$ & 6.6 & 6.6 & 20 \\
\hline 4 & $\left(<7^{\circ}, \sim 10^{\circ}\right)$ & 20 & 10 & 0 & 3.3 & 9.9 & 30 \\
\hline 5 & $\left(<7^{\circ}, \sim 15^{\circ}\right)$ & 20 & 30 & 0 & 3.2 & 16.3 & 50 \\
\hline 6 & $\left(\sim 35^{\circ},<7^{\circ}\right)$ & 50 & 70 & 180 & 34.7 & 4 & -20 \\
\hline 7 & $\left(\sim 25^{\circ},<7^{\circ}\right)$ & 50 & 30 & 180 & 25 & 5.6 & 20 \\
\hline 8 & $\left(<7^{\circ}, \sim 25^{\circ}\right)$ & 50 & 30 & 0 & 5.6 & 25.1 & 80 \\
\hline 9 & $\left(<7^{\circ}, \sim 35^{\circ}\right)$ & 50 & 70 & 0 & 4 & 34.7 & 120 \\
\hline
\end{tabular}

\section{A Proposal for Optimal Measurement Geometries}

The previous analysis suggests a strategy for selecting the measurement geometries for this kind of coating. We propose choosing those geometries required to characterize the functions $d\left(\theta_{\text {flake }}\right), a_{\text {low }, p}, a_{\text {high }, p}$, and $\left\langle f_{r, 0}\left(\theta_{i}, \phi_{i} ; \theta_{s}, \phi_{s}\right\rangle_{\lambda}\right.$. These geometries will explore thoroughly enough the variety of spectral distributions to obtain the corresponding spectral components by PCA. Therefore, our suggestion is to choose five measurement geometries at low $\theta_{\text {inc }}$ angles and welldistributed values of $\theta_{\text {flake }}$ [characterization of $d\left(\theta_{\text {flake }}\right)$, Fig. 6] and, symmetrically, five measurement geometries at low $\bar{\theta}_{\text {flake }}$ angles and well-distributed values of $\theta_{\text {inc }}$ (characterization of $a_{\text {low }, p}$ and $a_{\text {high }, p}$, Fig. 7). Since one of the geometries belongs to both groups (low $\theta_{\text {inc }}$ and $\theta_{\text {flake }}$ angles), the final number of selected measurement geometries amounts to nine. They are all shown in Table 1 , sorted by $\theta_{i}$ first and secondly by $\theta_{\text {asp }}$.

Comparison of these proposed measurement geometries with some available commercial instrument geometries (X-Rite MA98, Datacolor FX10, and BYK-mac) is shown in Table 2. The main difference between the proposed geometries lies in the definition of the low incidence angle aspecular line (these instruments lack 4 and 5 geometries). On the other hand, our analysis did not show the necessity of the usual commercial geometry $\left[\theta_{i}=45, \theta_{\text {asp }}=45^{\circ}\right]$. This classical geometry measures the color behavior of solid pigments (absorbents), which is redundant information, since it is already provided by geometries 8 and 9 in Table 1 .

Table 2. Comparison of the Measurement Geometries That We Propose with the Geometries of Those Instruments That Are Commercially Available

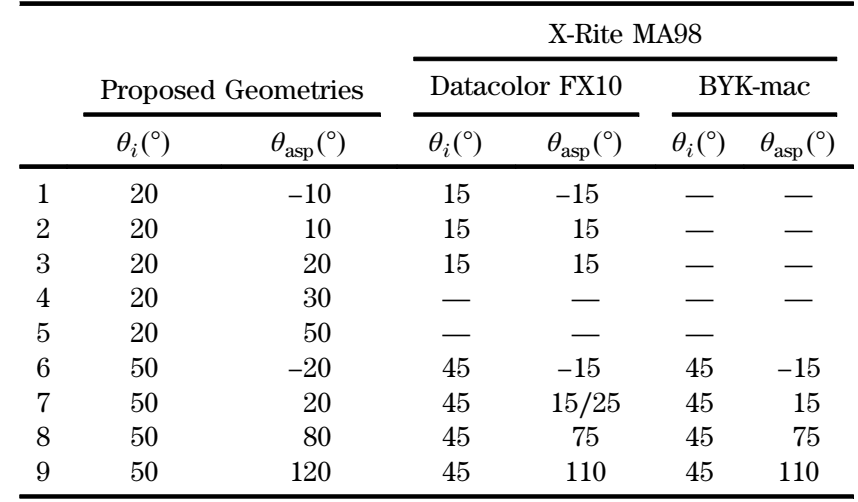

\section{DISCUSSION}

The proposed measurement geometries, which were selected following a rationale based on a physical model, can be regarded as two aspecular lines with well-separated incidence angles $\theta_{i}\left(20^{\circ}\right.$ and $\left.50^{\circ}\right)$. One of the practical advantages of these geometries is that future instruments would need to be designed only for these two irradiation angles and for seven viewing angles, which are symmetrical with respect to the normal $\left(0^{\circ}, \pm 10^{\circ}, \pm 30^{\circ}\right.$, and $\left.\pm 70^{\circ}\right)$. Nevertheless, the most important advantage is that the knowledge of the spectral BRDF for these geometries makes it possible to estimate the spectral reflectance (and color) for any other geometry. An algorithm based on the equations and conclusions of this work was designed for this particular task. The performance of this algorithm was assessed for two different special effect coatings: Colorstream Arctic Fire [25] and Colorstream Lapis Sunlight [26]. However, the mathematical approach (in the presented or in a similar form) is not restricted only to the studied coatings, but to the coatings whose spectral reflectance can be explained under the assumptions of the model presented in [19]. If it is not the case, the proper model should be applied in the analysis. The spectral BRDF of these two coatings was measured for the 448 geometries mentioned in Section 3, and the results were compared with the spectral BRDF estimated by the algorithm based on the nine proposed measurement geometries. Histograms of the color differences $\Delta E_{a b}$ (D65 illuminant, $2^{\circ}$ observer) between the measurement and the estimation for Arctic Fire and Lapis Sunlight are shown in Figs. 8 and 9 . Most of the differences are below 5 , while the highest differences are found for geometries were $\theta_{\text {flake }}$ is very small; that is, close to specular geometries.

These estimations would be hard to make relying on CIE $L^{*} a^{*} b^{*}$ diagrams since many measurement geometries should be used to obtain a clear characterization of the color shift. However, since the colorimetric representation is broadly used in the industry, the interpretation of the proposed geometries in CIE $L^{*} a^{*} b^{*}$ diagrams was examined.

The Arctic Fire experimental data discussed in this paper (Figs. 5-7) and the experimental data of Lapis Sunlight were plotted in CIE $L^{*} a^{*} b^{*}$ diagrams (Figs. 10 and 11). Large symbols represent in-plane measurements, whereas small, solid black circles represent out-of-plane measurements. In addition, the nine proposed geometry measurements are plotted as much bigger hollow circles. Aspecular lines corresponding to in-plane measurements are also plotted. After thorough inspection of these figures, some general conclusions can be drawn: 


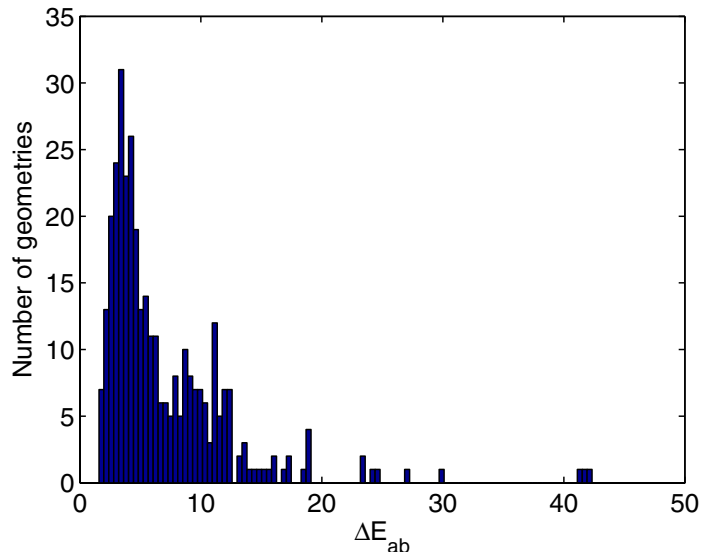

Fig. 8. (Color online) Histogram of color differences between the measurement and the estimation from the nine proposed geometries (Arctic Fire). The binning is 0.4 .

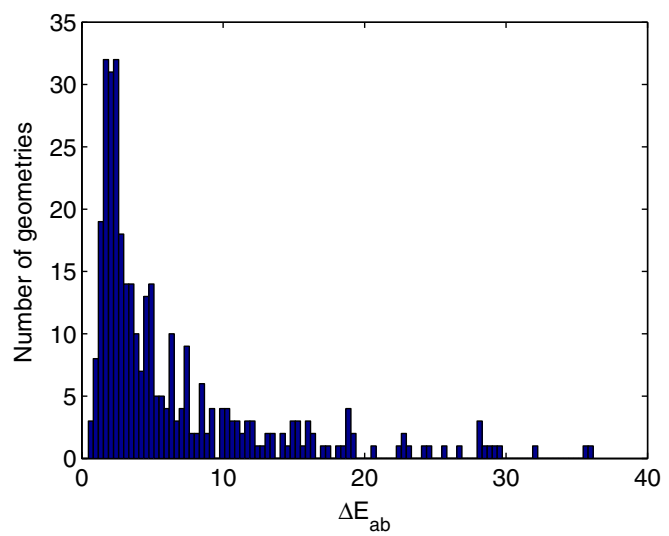

Fig. 9. (Color online) Histogram of color differences between the measurement and estimation from the nine proposed geometries (Lapis Sunlight). The binning is 0.35 .

1. Most of the points are concentrated around a color region (those of high aspecular angles): the base-coat color.

2. Aspecular lines flow out of the base-coat color when the aspecular angle decreases.
3. A well-defined grid can be distinguished. The logic behind this grid is Helmholtz reciprocity theorem (the results should be invariable with the interchange of $\theta_{i}$ and $\theta_{s}$ ), which can clearly be applied in this case: aspecular line $\theta_{i}$ cross aspecular line $\theta_{i}^{\prime}$ where its $\theta_{s}$ equals $\theta_{i}^{\prime}$. Another consequence of Helmholtz reciprocity theorem is that cis and trans interference lines overlap:

$$
\theta_{i} \operatorname{asp}\left(\theta_{\text {asp }}\right) \equiv\left(\theta_{i}-\theta_{\text {asp }}\right) \operatorname{asp}\left(-\theta_{\text {asp }}\right)
$$

1. As expected, the lower the aspecular angle, the higher the chroma.

2. Again, as expected, every aspecular line tends to a different hue, but not to a constant hue, since the hue changes with the aspecular angle as well. This is the reason why aspecular lines are curved even for low aspecular angles.

The results for the proposed measurement geometries are marked with big hollow circles in Figs. 10 and 11, labeled with their corresponding identification number included in Table 1 . Geometries from 1 to 5 may represent the measured aspecular line $\theta_{i}=20^{\circ}$ and geometries from 6 to 8 represent the measured aspecular line $\theta_{i}=50^{\circ}$. Due to its high aspecular angle, geometries 5,8 , and 9 define the base coat color area; for instance, the color gamut observed when the object is viewed under hemispherical illumination (outdoors). The difference of hue between geometries 3 and 4 (or 5) may represent the variation in hue when $\theta_{\text {inc }}$ is low (low $\theta_{i}$ and $\theta_{s}$ ), mostly due to the $\theta_{\text {flake }}$ variation. Such a situation occurs, for instance, when the object is illuminated and viewed from nearby directions. Finally, geometries 1, 2, 6, and 7 represent the highchroma color shift that makes these coatings so special. As a matter of fact, the color shift may be schematically represented by means of five arrows from: (1) $9\left(\theta_{i}=50^{\circ}\right.$ or $\left.70^{\circ}\right)$ to $3\left(\theta_{i}=0^{\circ}\right.$ or $\left.20^{\circ}\right)$; (2) 3 to $2\left(\theta_{i}=10^{\circ}\right.$ or $\left.20^{\circ}\right)$; (3) 2 to $1\left(\theta_{i}=20^{\circ}\right.$ or $\left.30^{\circ}\right)$; (4) 1 to $7\left(\theta_{i}=30^{\circ}\right.$ or $\left.50^{\circ}\right)$; and (5) 7 to $6\left(\theta_{i}=50^{\circ}\right.$ or $\left.70^{\circ}\right)$. Arctic Fire and Lapis Sun Light's color shift are plotted in Fig. 12 using this simple procedure.

These geometries can summarize the color of a goniochromatic coating. If, in addition, the above-mentioned

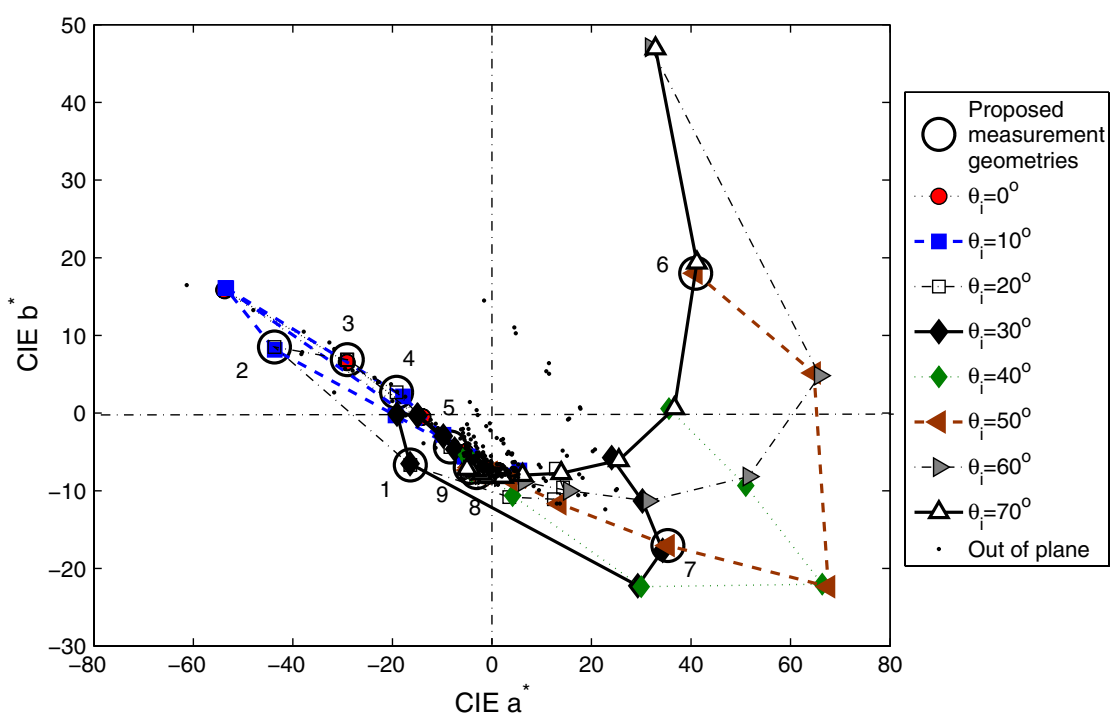

Fig. 10. (Color online) Color representation of Arctic Fire in CIE- $a^{*}, b^{*}$ coordinates. 


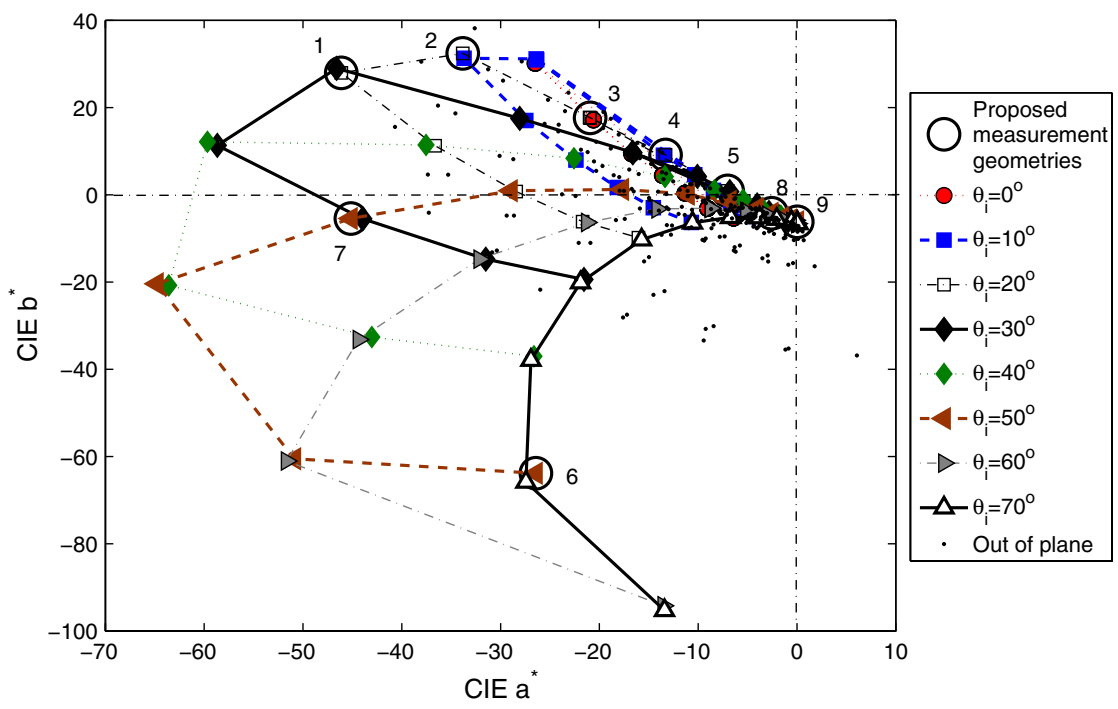

Fig. 11. (Color online) Color representation of Lapis SunLight in CIE- $a^{*}, b^{*}$ coordinates.

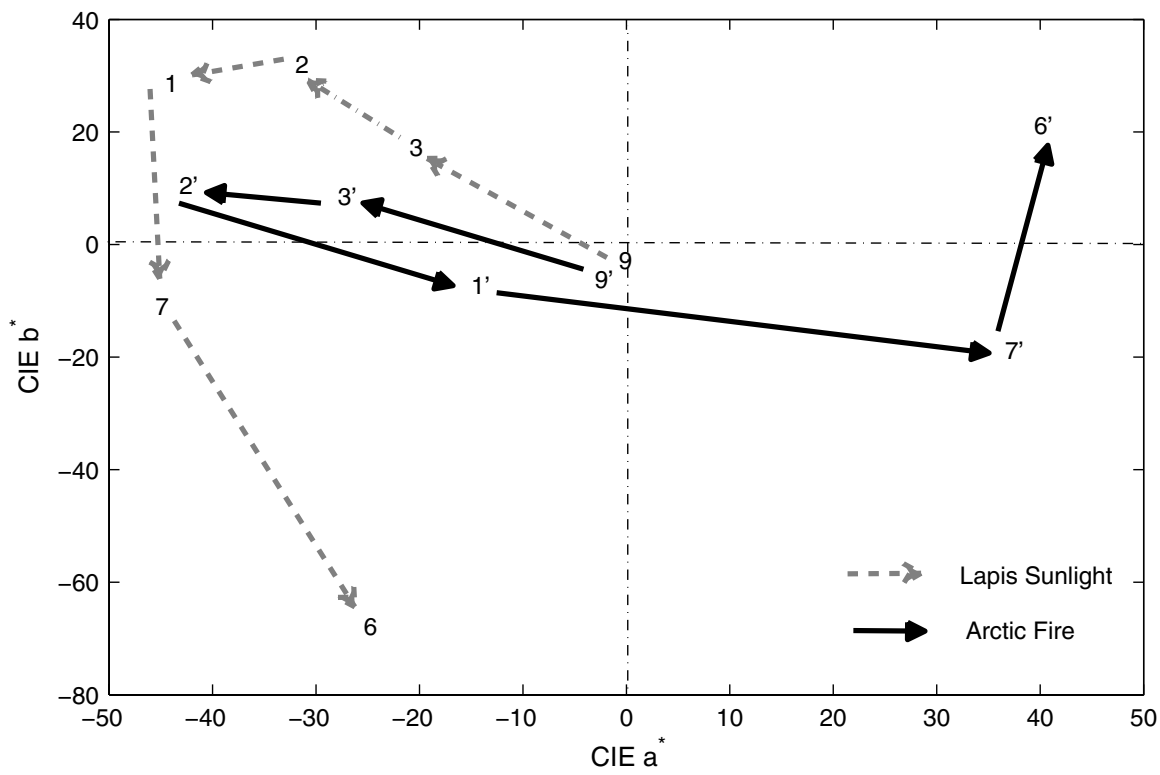

Fig. 12. Summary of color shifts for Arctic Fire and Lapis Sunlight.

conclusions about these coatings are borne in mind, a very complete description of the CIE $L^{*} a^{*} b^{*}$ diagram is obtained. Actually, if there is not an interest to obtain a description in terms of Eq. (15) but only in terms of the CIE $L^{*} a^{*} b^{*}$ diagram, low-chroma and high aspecular angle measurement geometries 5 and 8 are not required since the base-coat color can be well-defined by means of measurement geometry 9 .

\section{CONCLUSIONS}

A reduced set of nine measurements geometries has been proposed to characterize the spectral reflectance (and, consequently, the color) of special-effect coatings, so as to be able to infer their spectral reflectance for any other given geometry. The proposed geometries are not based on the analysis of a number of samples: A physical model based on flake-related parameters was used to determine which measurement geometries are redundant for the complete description of the spectral BRDF and which are the proper measurement geometries set for a good spectral BRDF sampling. These nine proposed geometries are easy to reproduce and should help manufactures to design instruments that would permit a more accurate characterization of the spectral reflectance of special-effect coatings.

The analysis of experimental spectral BRDF data was made without prior colorimetric simplification: we did not study the variation of colorimetric values, such as lightness, hue, or chroma across the geometries, but the variation of the set of BRDF spectra. In order to accomplish this multivariate task, we resorted to PCA, whose ability to extract this kind of data patterns had been demonstrated in previous works [20,21]. Using this technique in combination with the physical model, it is possible to estimate the color of a coating for any geometry from the measurements at a small number of proper geometries. 
This new methodology has been tested with two extreme special-effect coatings, not common yet in the current industrial applications. The studied coatings present an extreme colorimetric effect. It was shown that the proposed geometries provide a good estimation of the color shift. The estimation of the color might work much better for coloristically more simple effect pigments such as metallic and mica-based flake ones, which dominate most industrial applications of effect coatings and should be examined in a future work.

The measurement geometries proposed in this work can be regarded as two aspecular lines with well-separated illumination angles $\left(\theta_{i}\right)$. Therefore, future instruments could be designed only for these two illumination angles and for seven viewing angles that are symmetrical with respect to the surface normal of the sample plane. Nonetheless, the most important advantage is that these measurement geometries make it possible to estimate the color for other geometries.

Taking into account current commercially available multigonio-spectrophotometers, the measurement geometries implemented in X-Rite MA98 and Datacolor FX10 are actually closer to the ones proposed in this work than the geometries implemented in BYK-mac-which, on the other hand, unlike the above-mentioned instruments, allows sparkle and graininess to be measured. The experimental and mathematical methods presented in this work can be similarly used to assess how accurate the measurement geometries of these instruments allow the color at any other geometry to be estimated.

\section{ACKNOWLEDGMENTS}

The authors are grateful to "Plan Nacional de Física" for funding this work (FIS2010-19756-E), to CSIC's JAE Program, and the "European Social Fund" for awarding us a research trainee. This study was also supported by the Spanish Ministry of Economy and Competitiveness under the grant DPI201130090-C02-02 and the European Union. The authors have no interests in the development or marketing of any product mentioned in this study.

\section{REFERENCES}

1. F. J. Maile, G. Pfaff, and P. Reynders, "Effect pigments-past, present and future," Prog. Org. Coat. 54, 150-163 (2005).

2. H. J. Streitberger and K. F. Dössel, Automotive Paints and Coatings (Wiley-VCH, 2008).

3. M. E. Nadal and E. A. Early, "Color measurements for pearlescent coatings," Color Res. Appl. 29, 38-42 (2004).

4. G. Baba, "Gonio-spectrophotometric analysis of pearl-mica paint," Die Farbe 37, 99-110 (1990).

5. T. A. Germer and M. E. Nadal, "Modeling the appearance of special effect pigment coatings," Proc. SPIE 4447, 77-86 (2001).

6. M. E. Nadal and T. A. Germer, "Colorimetric characterization of pearlescent coatings,” Proc. SPIE 4421, 757-760 (2001).
7. B. Parker, "Color shift of light interference pigments," Surf. Coat. Aust. 39, 10-13 (2002).

8. F. Leloup, P. Hanselaer, M. Pointer, and J. Versluys, "Characterization of gonio-apparent colours," in Congress of the International Colour Association, 10th ed. (AIC, 2005), pp. $515-518$

9. C. A. Nicholls, "Visual and instrumental characterisation of special-effect colours," Ph.D. Thesis (University of Leeds, 2000).

10. R. Besold, "Metallic effect-characterization, parameter and methods for instrumentally determination," Die Farbe $\mathbf{3 7}$ 79-85 (1990).

11. "Tolerances for automotive paints, Part 2: Goniochromatic paints (Farbtoleranzen für Automobillackierungen-Teil 2: Effektlackierungen), DIN 6175-2" (Deutsche Institut für Normung e. V., 2001).

12. E. Chorro, E. Perales, F. M. Martínez-Verdú, J. Campos, and A Pons, "Colorimetric and spectral evaluation of the optical anisotropy of metallic and pearlescent samples," J. Mod. Opt. 56, 1457-1465 (2009).

13. E. Kirchner, G. J. Van den Kieboom, L. Njo, R. Supér, and R. Gottenbos, "Observation of visual texture of metallic and pearlescent materials," Color Res. Appl. 32, 256-266 (2007).

14. W. R. Cramer, "Reflections on the right angle," Eur. Coat. J. 4, 32-37 (2012).

15. E. Perales, E. Chorro, W. R. Cramer, and F. M. Martínez-Verdú, "Analysis of the colorimetric properties of goniochromatic colors using the MacAdam limits under different light sources," Appl. Opt. 50, 5271-5278 (2011).

16. F. E. Nicodemus, J. C. Richmond, and J. J. Hsia, "Geometrical considerations and nomenclature for reflectance," NBS Monogr. 160, 3-7 (1977).

17. A. Takagi, S. Sato, and G. Baba, "Prediction of spectral reflectance factor distribution of color-shift paint finishes," Color Res. Appl. 32, 378-387 (2007).

18. "Standard practice for multiangle color measurement of interference pigments," ASTM E 2539-08 (American Society for the Testing of Materials, 2009).

19. E. Kirchner and W. Cramer, "Making sense of measurement geometries for multi-angle spectrophotometers," Color Res. Appl. 37, 186-198 (2012).

20. A. Ferrero, J. Campos, A. M. Rabal, A. Pons, M. L. Hernanz, and A. Corróns, "Principal components analysis on the spectral bidirectional reflectance distribution function of ceramic colour standards," Opt. Express 19, 19199-19211 (2011).

21. A. Ferrero, J. Campos, A. M. Rabal, A. Pons, M. L. Hernanz, and A. Corróns, "Variables separation of the spectral BRDF for better understanding color variation in special effect pigment coatings," J. Opt. Soc. Am. A 29, 842-847 (2012).

22. A. M. Rabal, A. Ferrero, J. Campos, J. L. Fontecha, A. Pons, A. M. Rubiño, and A. Corróns, "Automatic gonio-spectrophotometer for the absolute measurement of the spectral BRDF at in \& out-of-plane and retroreflection geometries," Metrologia 49, 213-223 (2012)

23. G. Pfaff and P. Reynders, "Angle-dependent optical effects deriving from submicron structures of films and pigments," Chem. Rev. 99, 1963-1982 (1999).

24. E. J. J. Kirchner and J. Houweling, "Measuring flake orientation for metallic coatings," Prog. Org. Coat. 64, 287-293 (2009)

25. Colorstream T20-02 WNT Artic Fire, Product Information, Colorstream (Merck KgaA, 2004).

26. Colorstream T20-04 WNT Lapis Sunlight, Product Information, Colorstream (Merck KgaA, 2004). 